\title{
Sensor-fault Detection and Isolation using Interval Observers
}

\author{
Feng Xu, Vicenç Puig, Carlos Ocampo-Martinez, Florin Stoican, and Sorin Olaru
}

\begin{abstract}
This paper proposes an interval observer-based sensor fault detection and isolation (FDI) approach for the closed-loop systems. In this proposed approach, residuals are defined in such a way that their components are independent of each other at the time instant after fault occurrence, namely $k_{f}+1$, where $k_{f}$ denotes the fault occurrence time instant. In this way, it is guaranteed that at $k_{f}+1$ the changes in each component of the residuals are only related to the fault in the corresponding sensor. By detecting the threshold violation of the corresponding residual interval components, the proposed method can detect and isolate sensor faults at that time instant. At the end of this paper, a numerical example is used to present the effectiveness of the approach.
\end{abstract}

\section{INTRODUCTION}

Interval observers, appeared in the last decade, are a wellknown set-theoretic techniques for robust state estimation. Generally, they can estimate bounds for the system states and outputs enclosing the real ones at each time instant. This property is widely used for fault detection (FD) [2], [5].

The principle of the interval observer-based FD approach consists in propagating the effect of uncertainties by system mathematical models to generate interval vectors for real states and outputs at each time instant. Provided that the system is healthy, the current outputs should be inside their interval vectors estimated by the interval observer in real time. When the system is affected by a fault, the fault is indicated as long as the measured outputs take values out of their estimated interval vector.

However, regarding fault isolation (FI), less work has been done for the interval observer-based approach so far. In practical applications, in order to isolate faults, interval observers always turn to other techniques such as the fault signature matrix. In the previous work [6], we proposed to use a bank of interval observers for sensor FI. However, the FDI conditions needed were conservative and the computational resources required were quite demanding. Thus, the main motivation of this paper is to implement the interval observer-based FD and FI without the help of other existing FI techniques and improve the previous interval observerbased sensor FDI strategy proposed in [6]. Additionally, considering the balance among the expressional compactness, computational precision and complexity, zonotopes are

F. Xu, V. Puig and C. Ocampo-Martinez are with the Institut de Robòtica i Informàtica Industrial (CSIC-UPC), Technical University of Catalonia, Llorens i Artigas, 4-6, 08028 Barcelona, Spain, \{fxu, vpuig, cocampo\}@iri.upc.edu.

F. Stoican is with the Politehnica University of Bucharest (PUB) Department of Automatic Control and Systems Engineering, Bucharest, Romania, florin.stoican@acse.pub.ro.

S. Olaru is with the E3S (SUPELEC Systems Sceinces), Automatic Control Departement, Gif sur Yvette, France, sorin.olaru@supelec.fr. chosen as the containment set for uncertainties to propagate their effect on interval observers, referring to [1] for the applications of zonotopes in state estimation.

In this paper, the proposed interval observer-based sensor FDI is different from the classical FD use of interval observers. Thus, in order to target the core of the problem, the proposed approach is based on a closed-loop system scheme presented in Figure 1, which encompasses a linear discrete time-invariant plant, a reference system and a nominal interval observer. By specifically designing the residuals for the closed-loop system shown in Figure 1, faults in the sensor outputs can be effectively detected and isolated.

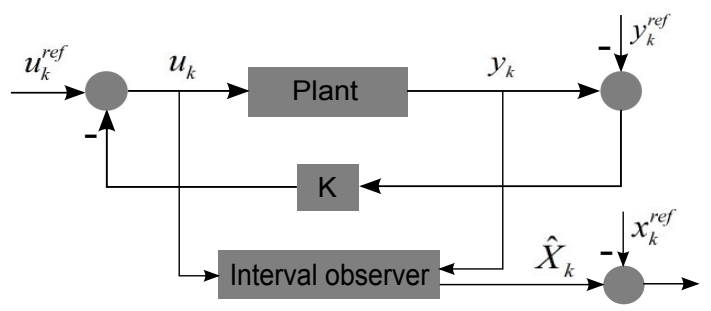

Fig. 1. Interval observer-based sensor FDI scheme

The main contribution of this paper is twofold. First, the proposed technique uses interval observers in a novel way to detect and isolate faults by testing whether residual intervals violate their bounds instead of detecting whether the zero vector is included by the residual intervals at each time instant. Second, the approach proposes a simple way to extend the use of interval observers into sensor FI and performs sensor FD and FI at the same time instant, which is also the advantage of the proposed method.

This paper is organized as follows. Section II introduces the notion of zonotopes and invariant sets. Section III introduces the closed-loop system shown in Figure 1. Section IV introduces the classical interval observer-based approach and the proposed interval observer-based FDI approach. In Section V, the type of faults that the proposed technique can detect and isolate are analyzed. In Section VI, a numerical example is used to illustrate the results. In Section VII, some conclusions are drawn.

\section{Preliminaries}

In this paper, the notation $\oplus$ represents the Minkowski sum of two sets, $|$.$| denotes the elementwise absolute value,$ $\mathbf{B}^{r}$ is a $r$-dimensional unitary box and the inequalities are interpreted elementwise. 


\section{A. Zonotopes}

According to [1] and [2], several definitions and properties related to zonotopes are introduced as follows.

Definition 2.1: The Minkowski sum of two sets $\mathrm{A}$ and $\mathrm{B}$ is defined by $A \oplus B=\{a+b: a \in A, b \in B\}$.

Definition 2.2: Given a vector $p \in \mathbb{R}^{n}$ and a matrix $G \in \mathbb{R}^{n \times m}(n \leq m)$, a zonotope $X$ with order $m$ is defined as $X=p \oplus G \mathbf{B}^{m 1}$.

Definition 2.3: The interval hull $\square X$ of a zonotope $X=p \oplus G \mathbf{B}^{r} \subset \mathbb{R}^{n}$ is the smallest interval box that contains $X$, i.e., $\square X=\left\{x:\left|x_{i}-p_{i}\right| \leq\left\|G_{i}\right\|_{1}\right\}$, where $G_{i}$ is the $i$-th row of $G$, and $x_{i}$ and $p_{i}$ are the $i$-th components of $x$ and $p$, respectively.

Property 2.1: Given zonotopes $X_{1}=p_{1} \oplus G_{1} \mathbf{B}^{r_{1}} \subset \mathbb{R}^{n}$ and $X_{2}=p_{2} \oplus G_{2} \mathbf{B}^{r_{2}} \subset \mathbb{R}^{n}$, the Minkowski sum of them is $X_{1} \oplus X_{2}=\left\{p_{1}+p_{2}\right\} \oplus\left[\begin{array}{ll}G_{1} & G_{2}\end{array}\right] \mathbf{B}^{r_{1}+r_{2}}$.

Property 2.2: Given a zonotope $X=p \oplus G \mathbf{B}^{r} \subset \mathbb{R}^{n}$ and a compatible matrix $K$, the image of the zonotope by the matrix is computed as $K X=K p \oplus K G \mathbf{B}^{r}$.

Property 2.3: Given a zonotope $X=p \oplus G \mathbf{B}^{r} \subset \mathbb{R}^{n}$ and an integer $s$ (with $n<s<r$ ), denote by $\hat{G}$ the matrix resulting from the recording of the columns of the matrix $G$ in decreasing Euclidean norm. $X \subseteq p \oplus\left[\begin{array}{ll}\hat{G}_{T} & Q\end{array}\right] \mathbf{B}^{s}$ where $\hat{G}_{T}$ is obtained from the first $s-n$ columns of matrix $\hat{G}$ and $Q \in \mathbb{R}^{n \times n}$ is a diagonal matrix whose elements satisfy $Q_{i i}=\sum_{j=s-n+1}^{r}\left|\hat{G}_{i j}\right|, i=1, \ldots, n$.

\section{B. Invariant Sets}

The linear discrete time-invariant dynamics

$$
x_{k+1}=A_{\circ} x_{k}+B_{\circ} \delta_{k}
$$

is used to present the invariant set notions, where $A_{\circ}$ and $B_{\circ}$ are constant matrices and $A_{\circ}$ is a Schur matrix, $\delta_{k}$ belongs to $\Delta=\left\{\delta:\left|\delta-\delta^{\circ}\right| \leq \bar{\delta}\right\}$ with $\delta^{\circ}$ and $\bar{\delta}$ constant.

Definition 2.4: A set $X \subset \mathbb{R}^{n}$ is called a robust positively invariant (RPI) set for (1) if and only if $A_{\circ} X \oplus B_{\circ} \Delta \subseteq X . \nabla$

Definition 2.5: The minimal RPI (mRPI) set of (1) is defined as a RPI set contained in any closed RPI set and the mRPI set is unique and compact.

Theorem 2.1: [4] Considering (1) and letting $A_{\circ}=$ $V \Lambda V^{-1}$ be the Jordan decomposition of $A_{\circ}$,

$$
\begin{aligned}
\Phi(\theta)= & \left\{x \in \mathbb{R}^{n}:\left|V^{-1} x\right| \leq(I-|\Lambda|)^{-1}\left|V^{-1} B_{\circ}\right| \bar{\delta}\right. \\
& +\theta\} \oplus \xi^{\circ}
\end{aligned}
$$

is RPI and attractive for the trajectories of (1), with $\theta$ any (arbitrarily small) vector with positive components, where $\xi^{\circ}$ is the center of the set with $\xi^{\circ}=\left(I-A_{\circ}\right)^{-1} B_{\circ} \delta^{\circ}$, where $I$ is the compatible identity matrix.

1) For any $\theta$, the set $\Phi(\theta)$ is (positively) invariant, that is, if $x_{0} \in \Phi(\theta)$, then $x_{k} \in \Phi(\theta)$ for all $k \geq 0$.

2) Given $\theta \in \mathbb{R}^{n}, \theta>0$, and $x_{0} \in \mathbb{R}^{n}$, there exists $k^{*} \geq 0$ such that $x_{k} \in \Phi(\theta)$ for all $k \geq k^{*}$.

${ }^{1}$ In this paper, $p$ and $G$ are called the center and segment matrix of the zonotope, respectively.
Proposition 2.1: [4] Considering (1) and denoting $X_{0}$ as a RPI initial set of (1), each of the set iterations

$$
X_{j+1}=A_{\circ} X_{j} \oplus B_{\circ} \Delta, \quad j \in \mathbb{N},
$$

where $j$ denotes the $j$-th element of the set sequence and $\mathbb{N}$ represents the set of natural numbers, is a RPI approximation of the mRPI set. Furthermore, as $j$ tends to infinity, the set sequence converges to the mRPI set.

\section{Plant and Interval ObServers}

\section{A. Plant and Reference System}

The linear discrete time-invariant plant is modeled as

$$
\begin{aligned}
x_{k+1} & =A x_{k}+B u_{k}+\omega_{k}, \\
y_{k} & =G_{i} C x_{k}+\eta_{k},
\end{aligned}
$$

where $A \in \mathbb{R}^{n \times n}, B \in \mathbb{R}^{n \times p}$ and $C \in \mathbb{R}^{q \times n}$ are constant parameter matrices, $x_{k} \in \mathbb{R}^{n}, u_{k} \in \mathbb{R}^{p}$ and $y_{k} \in \mathbb{R}^{q}$ are states, inputs and outputs, respectively, $\omega_{k}$ and $\eta_{k}$ represent system uncertainties in states and outputs (including disturbances, noises, offsets, etc), respectively, $k$ denotes the $k$-th discrete time instant and $G_{i}(i \in \mathbb{I}=\{0,1, \cdots, N\})$ is a $q \times q$ diagonal matrix modeling the sensor-fault situations, where $N$ denotes the number of considered sensor faults and $G_{0}$, the identity matrix, represents that all sensors are healthy ${ }^{2}$.

All diagonal elements of $G_{i}$ belong to $[0,1]$, where 0 and 1 represent the complete outage and health of the corresponding sensors, respectively, and a value in $(0,1)$ denotes partial performance degradation of the corresponding sensors. $\omega_{k}$ and $\eta_{k}$ are defined to belong to

$$
\begin{aligned}
W & =\left\{\omega_{k} \in \mathbb{R}^{n}:\left|\omega_{k}-\omega^{c}\right| \leq \bar{\omega}, \omega^{c} \in \mathbb{R}^{n}, \bar{\omega} \in \mathbb{R}^{n}\right\}, \\
V & =\left\{\eta_{k} \in \mathbb{R}^{q}:\left|\eta_{k}-\eta^{c}\right| \leq \bar{\eta}, \eta^{c} \in \mathbb{R}^{q}, \bar{\eta} \in \mathbb{R}^{q}\right\},
\end{aligned}
$$

where $\omega^{c}, \eta^{c}, \bar{\omega}$ and $\bar{\eta}$ are constant vectors. $W$ and $V$ can be also rewritten as zonotopes

$$
\begin{aligned}
W & =\omega^{c} \oplus H_{\bar{\omega}} \mathbf{B}^{n}, \\
V & =\eta^{c} \oplus H_{\bar{\eta}} \mathbf{B}^{q},
\end{aligned}
$$

where $H_{\bar{\omega}} \in \mathbb{R}^{n \times n}$ and $H_{\bar{\eta}} \in \mathbb{R}^{q \times q}$ are diagonal matrices with the diagonal entries $\bar{\omega}$ and $\bar{\eta}$, respectively.

As shown in Figure 1, a reference system is designed for the system, whose dynamics is given by

$$
\begin{aligned}
x_{k+1}^{\mathrm{ref}} & =A x_{k}^{\mathrm{ref}}+B u_{k}^{\mathrm{ref}}, \\
y_{k}^{\mathrm{ref}} & =C x_{k}^{\mathrm{ref}} .
\end{aligned}
$$

Assumption 3.1: The reference inputs $u_{k}^{\text {ref }}$ belong to $U_{\text {ref }}=\left\{u_{k}^{\text {ref }} \in \mathbb{R}^{p}:\left|u_{k}^{\text {ref }}-u_{\text {ref }}^{c}\right| \leq \bar{u}_{\text {ref }}, u_{\text {ref }}^{c} \in \mathbb{R}^{p}, \bar{u}_{\text {ref }} \in\right.$ $\left.\mathbb{R}^{p}\right\}$, where $u_{\text {ref }}^{c}$ and $\bar{u}_{\text {ref }}$ are constant and, $U_{\text {ref }}$ is rewritten as a zonotope $U=u_{\text {ref }}^{c} \oplus H_{\bar{u}_{\text {ref }}} \mathbb{B}^{p}$, where $H_{\bar{u}_{\text {ref }}} \in \mathbb{R}^{p \times p}$ is a diagonal matrix with diagonal entries from $\bar{u}_{\text {ref }}$.

The control objective of closed-loop system is to asymptotically track the reference model outputs, i.e,

$$
\lim _{k \rightarrow \infty} y_{k}-y_{k}^{\text {ref }} \rightarrow 0 \text {. }
$$

\footnotetext{
${ }^{2}$ The number of rows in $C$ represents that of sensors installed in the system (each row corresponds to a sensor) and the status of the $i$-th sensor is modeled by the numeric value of the $i$-th diagonal element of $G$.
} 
Thus, the control law is designed as

$$
u_{k}=u_{k}^{\mathrm{ref}}-K\left(y_{k}-y_{k}^{\mathrm{ref}}\right),
$$

where $K$ is the feedback gain used to implement the control objective $^{3}$. According to (2), (5) and (6), the closed-loop dynamics of the system are derived as

$$
\begin{aligned}
x_{k+1}= & \left(A-B K G_{i} C\right) x_{k}+B u_{k}^{\mathrm{ref}}+B K y_{k}^{\mathrm{ref}} \\
& -B K \eta_{k}+\omega_{k}, \\
y_{k}= & G_{i} C x_{k}+\eta_{k},
\end{aligned}
$$

Assumption 3.2: The closed-loop system (7a) can be stabilized by the feedback gain matrix $K$, i.e., $A-B K G_{i} C$ in (7a) is a Schur matrix for all $G_{i}$.

\section{B. Interval Observers}

According to [2] and (2), the nominal interval observer is designed as

$$
\begin{aligned}
\hat{X}_{k+1}= & (A-L C) \hat{X}_{k} \oplus\left\{B u_{k}\right\} \oplus\left\{L y_{k}\right\} \\
& \oplus(-L) V \oplus W, \\
\hat{Y}_{k}= & C \hat{X}_{k} \oplus V,
\end{aligned}
$$

where $\hat{X}_{k}$ and $\hat{Y}_{k}$ are estimated state and output zonotopes at time instant $k$, respectively,

Assumption 3.3: The interval observer gain $L$ is chosen to ensure the contractiveness of the set mapping in (8).

According to (8) and the properties of zonotopes, the center $\hat{x}_{k+1}^{c}$ and segment matrix $\hat{H}_{k+1}^{x}$ of $\hat{X}_{k+1}$, and the center $\hat{y}_{k}^{c}$ and segment matrix $\hat{H}_{k}^{y}$ of $\hat{Y}_{k}$ are computed as

$$
\begin{aligned}
\hat{x}_{k+1}^{c} & =(A-L C) \hat{x}_{k}^{c}+B u_{k}+L y_{k}-L \eta^{c}+w^{c}, \\
\hat{H}_{k+1}^{x} & =\left[\begin{array}{ll}
(A-L C) \hat{H}_{k}^{x}-L H_{\bar{\eta}} & H_{\bar{\omega}}
\end{array}\right], \\
\hat{y}_{k}^{c} & =C \hat{x}_{k}^{c}+\eta^{c}, \\
\hat{H}_{k}^{y} & =\left[\begin{array}{ll}
C \hat{H}_{k}^{x} & H_{\bar{\eta}}
\end{array}\right] .
\end{aligned}
$$

Assumption 3.4: The initial state of the plant is represented as $x_{0}$, the initial zonotope of the nominal interval observer is denoted as $\hat{X}_{0}$ and $x_{0} \in \hat{X}_{0}$ holds.

\section{Classical Interval Observer-based FD}

Generally, the interval observer-based FD approach consists in testing if the real outputs of system are consistent with their corresponding predictions estimated by the interval observer at each time instant

$$
\mathbf{0} \in \square R_{k},
$$

where $R_{k}$ denotes residual zonotope defined as

$$
R_{k}=\left\{y_{k}\right\} \oplus\left(-\hat{Y}_{k}\right) .
$$

Remark 3.1: The interval hull is computed by Definition 2.3 and the order of zonotopes predicted by the interval observer is controlled by Property 2.3.

\footnotetext{
${ }^{3}$ The feedback gain can be designed by different control techniques such as pole placement or LQR.
}

In real-time testing, if (10) does not hold, it indicates that a fault is detected at time instant $k$. Otherwise, the system is still assumed to be in healthy situation.

One should note that, despite the interval observer-based approach can detect faults in this classical way of defining residuals as seen in (10), it is difficult to isolate faults by the same residual definition.

Thus, in order to implement the interval observer-based sensor FI, a novel way to define the residuals by turning to a reference system is proposed in this paper. Based on this novel residual definition, the interval observer-based approach can be extended to the case of sensor FI. The technical details will be elaborated in next sections.

Since interval vectors are computed by interval hulls of zonotopes, for simplicity, the following discussions are directly based on zonotopes.

\section{INTERVAL OBSERVER-BASED SENSOR FDI}

\section{A. Relevant Zonotopes}

In order to define proper residuals for the proposed technique, one considers

$$
\begin{aligned}
\tilde{X}_{k}^{\mathrm{ref}} & =\left\{x_{k}^{\mathrm{ref}}\right\} \oplus\left(-\hat{X}_{k}\right) \\
& =\left\{x_{k}^{\mathrm{ref}}-\hat{x}_{k}^{c}\right\} \oplus \hat{H}_{k}^{x} \mathbf{B}^{s_{k}},
\end{aligned}
$$

where $s_{k}$ denotes the order of $\hat{X}_{k}$ and $\tilde{X}_{k}^{\text {ref }}$, and $x_{k}^{\text {ref }}-\hat{x}_{k}^{c}$ and $\hat{H}_{k}^{x}$ are notated by $\tilde{x}_{k}^{c}$ and $\tilde{H}_{k}^{\tilde{x}}$, respectively.

According to (5), (6), (9) and (12), the center $\tilde{x}_{k+1}^{c}$ and segment matrix $\tilde{H}_{k+1}^{\tilde{x}}$ of $\tilde{X}_{k+1}^{\text {ref }}$ are derived as

$$
\begin{aligned}
\tilde{x}_{k+1}^{c}= & (A-B K C) \tilde{x}_{k}^{c}+(B K-L) G_{i} C x_{k}+(B K-L) \eta_{k} \\
& -(B K-L) C \hat{x}_{k}^{c}+L \eta^{c}-\omega^{c}, \\
\tilde{H}_{k+1}^{\tilde{x}}= & \hat{H}_{k+1}^{x}=\left[(A-L C) \hat{H}_{k}^{x}-L H_{\bar{\eta}} H_{\bar{\omega}}\right] .
\end{aligned}
$$

In the healthy situation, i.e., $G_{i}=G_{0}$ in (13), $\tilde{X}_{k+1}^{\text {ref }}$ is denoted as $\tilde{X}_{k+1}^{0, \text { ref }}$, whose expression is transformed into

$$
\begin{aligned}
\tilde{x}_{k+1}^{0, c}= & (A-B K C) \tilde{x}_{k}^{0, c}+(B K-L) C\left(x_{k}-\hat{x}_{k}^{c}\right) \\
& +(B K-L) \eta_{k}+L \eta^{c}-\omega^{c} \\
\tilde{H}_{k+1}^{0, \tilde{x}}= & \hat{H}_{k+1}^{x}=\left[(A-L C) \hat{H}_{k}^{x}-L H_{\bar{\eta}} H_{\bar{\omega}}\right]
\end{aligned}
$$

where $\tilde{x}_{k+1}^{0, c}$ and $\tilde{H}_{k+1}^{0, \tilde{x}}$ are the center and segment matrix of $\tilde{X}_{k+1}^{0 \text {,ref }}$, respectively. In order to describe $x_{k}-\hat{x}_{k}^{c}$ in the second summand (14a), a signal $\tilde{\tilde{x}}_{k}^{c}$ is defined as

$$
\tilde{\tilde{x}}_{k}^{c}=x_{k}-\hat{x}_{k}^{c}
$$

and in the healthy situation $\tilde{\tilde{x}}_{k}^{c}$ is denoted as $\tilde{\tilde{x}}_{k}^{0, c}$. Furthermore, according to (2) and (9a), the dynamics of $\tilde{\tilde{x}}_{k}^{0, c}$ is derived as

$$
\tilde{\tilde{x}}_{k+1}^{0, c}=(A-L C) \tilde{\tilde{x}}_{k}^{0, c}-L\left(\eta_{k}-\eta^{c}\right)+\left(\omega_{k}-\omega^{c}\right) .
$$

According to Theorem 2.1, Proposition 2.1 and the bounds of $\eta_{k}$ and $\omega_{k}(W$ and $V)$, an invariant set of $\tilde{\tilde{x}}_{k}^{0, c}$ described in (15) can be computed, which is denoted as $\tilde{\tilde{X}}^{0}$. According to (3) and (4), it is known that both $W$ and $V$ are zonotopes, thus, $\tilde{\tilde{X}}^{0}$ is also a zonotope whose center and segment matrix 
are notated as $\tilde{\tilde{x}}^{0, c}$ and $\tilde{\tilde{\tilde{H}}}^{0, x}$, respectively. One should note that the invariant set $\tilde{\tilde{X}}^{0}$ provides the bound for $\tilde{\tilde{x}}_{k}^{0, c}$ when the system is in the healthy functioning.

By substituting $\tilde{\tilde{X}}^{0}$ and $V$ into (14a) to respectively replace $x_{k}-\hat{x}_{k}^{c}$ and $\eta_{k}$ and using the zonotope operations, one can obtain a bounding zonotope denoted as $\tilde{X}_{k+1}^{0, \text { ref }}$ that bounds $\tilde{X}_{k+1}^{0, \text { ref }}$. Furthermore, according to (14), the center $\check{x}_{k+1}^{0, c}$ and segment matrix $\check{H}_{k+1}^{0, \tilde{x}}$ of $\check{X}_{k+1}^{0, \text { ref }}$ are derived as

$$
\begin{aligned}
\check{x}_{k+1}^{0, c}= & (A-B K C) \check{x}_{k}^{0, c}+(B K-L) C \tilde{\tilde{x}}^{0, c} \\
& +B K \eta^{c}-\omega^{c}, \\
\bar{H}_{k+1}^{0, \tilde{x}}= & (A-B K C) \bar{H}_{k}^{0, \tilde{x}}(B K-L) C \tilde{\tilde{H}}^{0, x} \\
& \left.(B K-L) H_{\bar{\eta}}\right], \\
\check{H}_{k+1}^{0, \tilde{x}}= & {\left[\bar{H}_{k+1}^{0, \tilde{x}}(A-L C) \hat{H}_{k}^{x}-L H_{\bar{\eta}} H_{\bar{\omega}}\right], }
\end{aligned}
$$

Assumption 4.1: In the healthy functioning, the initial zonotope $\tilde{X}_{0}^{0, \text { ref }}$ of (14) and the initial zonotope $\check{X}_{0}^{0 \text {,ref }}$ of (16) satisfy the condition that $\tilde{X}_{0}^{0 \text {,ref }} \subseteq \check{X}_{0}^{0 \text {,ref }}$.

Remark 4.1: As long as Assumption 4.1 is satisfied and the system is healthy, the inclusion $\tilde{X}_{k}^{0 \text {,ref }} \subseteq \check{X}_{k}^{0 \text {,ref }}$ always holds for $k>0$.

\section{B. Proposed Interval Observer-based Sensor FDI}

According to (13), it is shown that sensor faults impact $\tilde{X}_{k}^{\text {ref }}$ through the term $(B K-L) G_{i} C x_{k}$. In order to decouple the impact of different faults on different residual components, one turns to the Moore-Penrose inverse.

Property 4.1: Given a matrix $F \in \mathbb{C}^{m \times n}$ and its MoorePenrose inverse $F^{+} \in \mathbb{C}^{n \times m}$, it is concluded that

- $F F^{+}=I_{m} \Leftrightarrow \operatorname{rank}(F)=m$;

- $F^{+} F=I_{n} \Leftrightarrow \operatorname{rank}(F)=n$,

where $I_{m}$ and $I_{n}$ denote the identity matrices with the dimension $m \times m$ and $n \times n$, respectively.

In (13), it is known that $B K-L$ is a $n \times q$ matrix whose Moore-Penrose inverse is denoted as $(B K-L)^{+}$ with the dimension $q \times n$. In the structure of $B K-L$, it is known that both $K$ and $L$ are adjustable matrices, i.e., designing flexibility, which are determined by the designers. Thus, according to Property $4.1, K$ and $L$ can be properly selected such that $\operatorname{rank}(B K-L)=q$ holds.

Assumption 4.2: For the system (2), the number of sensors are not larger than that of system states, i.e., $q \leq n$.

Remark 4.2: As long as Assumption 4.2 is satisfied, and $K$ and $L$ ensure $\operatorname{rank}(B K-L)=q$, according to Property 4.1 , one has

$$
(B K-L)^{+}(B K-L)=I_{q},
$$

where $I_{q}$ is the identity matrix with the dimension $q \times q$. $\diamond$

In virtue of the aforementioned discussions, the residual for this proposed sensor FDI approach is defined as

$$
R_{k}=(B K-L)^{+} \tilde{X}_{k}^{\text {ref }},
$$

where $R_{k}$ denotes the residual zonotope at time instant $k$, whose center and segment matrix are denoted as $r_{k}^{c}$ and $H_{k}^{r}$, respectively. Furthermore, according to (13) and (17), the center $r_{k+1}^{c}$ and segment matrix $H_{k+1}^{r}$ of the residual zonotope $R_{k+1}$ at time instant $k+1$ are derived as

$$
\begin{aligned}
r_{k+1}^{c}= & (B K-L)^{+}(A-B K C) \tilde{x}_{k}^{c}+G_{i} C x_{k}-C \hat{x}_{k}^{c}+\eta_{k} \\
& +(B K-L)^{+} L \eta^{c}-(B K-L)^{+} \omega^{c}, \\
H_{k+1}^{r}= & (B K-L)^{+}(A-L C) \hat{H}_{k}^{x}-(B K-L)^{+} L H_{\bar{\eta}} \\
& \left.(B K-L)^{+} H_{\bar{\omega}}\right],
\end{aligned}
$$

It is seen that sensor faults do not impact the segment matrix (or size ${ }^{4}$ ) of the residual zonotopes as described in (19b) but affect the center as described in (19a) through the term $G_{i} C x_{k}$.

It is assumed that a fault in the $i$-th sensor occurs at time instant $k$. According to the structure of $G_{i} C x_{k}$ in (19a), at $k$ the fault only impacts the $i$-th element of $G_{i} C x_{k}$ and does not affect its other elements. Further, it means that the fault effect on the $i$-th element of $G_{i} C x_{k}$ at $k$ will be transmitted into the $i$-th element of $r_{k+1}^{c}$ at time instant $k+1$ but will not affect any of the other elements of $r_{k+1}^{c}$. It implies that at $k+$ 1 only the $i$-th component of $R_{k+1}$ contains the information of the fault in the $i$-th sensor and the other components of $R_{k+1}$ will not be affected by the fault, which provides a way to detect and isolate the sensor fault at the same time instant.

In order to detect and isolate sensor faults accurately, a threshold for the residual zonotopes $R_{k}$ should be generated. Under Assumption 4.1 and according to (16), it is known that $\check{X}_{k+1}^{0, \text { ref }}$ always bounds $\tilde{X}_{k+1}^{0 \text {,ref }}$, thus, the threshold of $R_{k}$ is defined as

$$
\check{R}_{k}=(B K-L)^{+} \check{X}_{k+1}^{0, \text { ref }} .
$$

When the system is healthy, $\check{R}_{k}$ computed by (20) will always bound $R_{k}$ computed by (18), thus, the detection and isolation of sensor faults consist in testing the relationships:

$$
\square R_{k}^{i} \subset \square \check{R}_{k}^{i}, \quad i=1,2, \cdots, q,
$$

where $\square R_{k}^{i}$ and $\square \check{R}_{k}^{i}$ represent the $i$-th component of $\square R_{k}$ and $\square \check{R}_{k}$, respectively.

In practical testing, if the $i$-th relationship in (21) corresponding to the $i$-th sensor is violated, the $i$-th sensor is marked as faulty at this time instant, otherwise the sensor is still assumed to be healthy. In order to clarify the proposed sensor FDI approach, a procedure is described by Algorithm 1.

\section{Detectable and Isolable Faults}

According to [3], for interval observers, faults are classified into three types: non-detectable faults, permanently detected faults and non-permanently detected faults. This fault classification is done according to the properties of the faults. In the proposed sensor FDI approach, the detection and isolation of faults are done at the same time whenever the corresponding elementwise relationships in (21) are violated, where similarly whether or not (21) is violated relies on the properties of the faults.

\footnotetext{
${ }^{4}$ The size of a zonotope corresponds to of its interval hull width.
} 


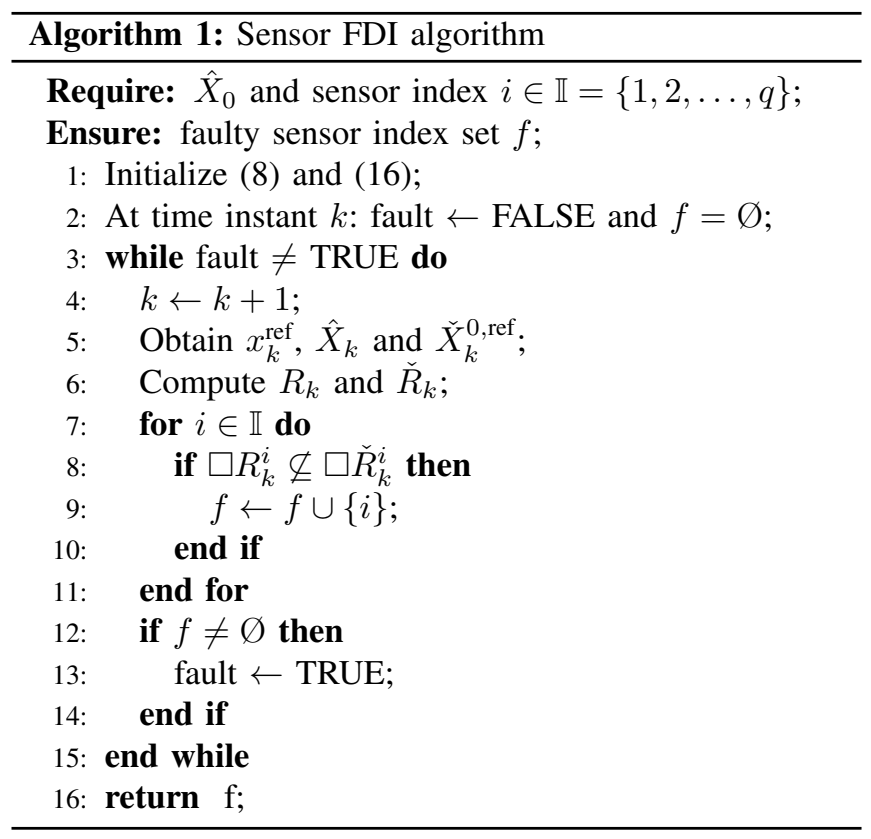

According to (19a), this technique can guarantee reliable FI in case that the faults violate their corresponding elementwise relationships one step after their occurrence, i.e., at time instant $k_{f}+1$. However, after more than one step, (i.e., $k>k_{f}+1$ ) all the components of residual zonotopes will be affected by the faults that occur in those sensors (as seen in (19a)). This implies that the effect of the faults on the components of residual zonotopes can not be completely decoupled from each other, i.e, a fault appearing in one sensor may result in the violation of the components corresponding to the other sensors. In light of this point, when using this method to detect and isolate faults, one faces three different possibilities:

- a fault occurs but Algorithm 1 can not detect the violation of (21). It means the fault is not detectable or isolable by the algorithm. This case is related to the fault sensitivity of interval observers and is inherent to the interval observer-based approach;

- a fault occurs and the elementwise relationship corresponding to the fault in (21) is violated one step after the fault occurrence. In this case, the fault can be detected and isolated accurately. This type of faults is the main target Algorithm 1 wants to detect and isolate;

- a fault occurs and the elementwise relationship corresponding to the fault in (21) is violated more than one step after the fault occurrence. In this case, since the accurate FI condition aforementioned does not exist, Algorithm 1 can only isolate a set of sensors where the faulty sensor is included. However, the set of sensors perhaps also include some healthy sensors.

Remark 5.1: Regarding the third type of faults, it is still possible for Algorithm 1 to accurately isolate the faults but it depends on the fault properties. The worst case for the isolation of these faults is that Algorithm 1 considers that all the sensors are faulty, which implies that Algorithm 1 may not be able to accurately isolate the faulty sensors, but at least it can reliably isolate all of them.

According to the discussions, it is known that the proposed method can accurately and reliably isolate the second type of faults, and reliably but conservatively isolate the third type of faults. In order to improve its effectiveness and reliability, in practical applications one takes the following measures:

- only trust the earliest diagnostic alerts provided by Algorithm 1 and ignore all its diagnostic results at the following time instants,

- when one and only one elementwise relationship in (21) is violated, Algorithm 1 decides that the corresponding sensor is faulty,

- when more than one elementwise relationships in (21) are violated, Algorithm 1 decides that all the corresponding sensors are faulty, despite some of them may be still healthy.

Note that the sensor faults generally affect the system outputs quickly after their occurrence. Thus, in general, this proposed technique should be useful for sensor FDI.

\section{NUMERICAL EXAMPLE}

A numerical example with two sensors is considered, whose dynamics are written as in (2) and $i \in\{0,1,2\}$ is the index of the sensor situations, where 0 represent the health situation.

The nominal interval observer is designed as (8) and a reference system is designed as (5). Besides, the residual zonotope and its threshold are defined as (18) and (20). The parameters of the numerical example are given as

- model parameters:

$$
\begin{aligned}
A & =\left[\begin{array}{cc}
0.8667 & -1.2343 \\
0.01 & 1
\end{array}\right], B=\left[\begin{array}{cc}
0.01 & 1 \\
1 & 0.01
\end{array}\right], \\
C & =\left[\begin{array}{cc}
0.5 & 0 \\
0 & 1.5
\end{array}\right],
\end{aligned}
$$

- process disturbances: $\bar{w}=\left[\begin{array}{l}0.1 \\ 0.1\end{array}\right], w^{c}=\left[\begin{array}{l}0 \\ 0\end{array}\right]$,

- measurement noises: $\bar{\eta}=\left[\begin{array}{l}0.05 \\ 0.05\end{array}\right], \eta^{c}=\left[\begin{array}{l}0.15 \\ 0.15\end{array}\right]$,

- observer gain: $L=\left[\begin{array}{cc}0.3334 & -0.8229 \\ 0.02 & 0.1333\end{array}\right]$,

- feedback gain: $K=\left[\begin{array}{cc}0.0107 & 0.3111 \\ 1.0248 & -0.8263\end{array}\right]$,

- fault magnitude: $G_{1}=\left[\begin{array}{cc}0.8 & 0 \\ 0 & 1\end{array}\right], G_{2}=\left[\begin{array}{cc}1 & 0 \\ 0 & 0.5\end{array}\right]$,

- reference input (sinusoidal signal) set:

$$
U_{\text {ref }}=\left[\begin{array}{l}
2 \\
2
\end{array}\right] \oplus\left[\begin{array}{cc}
0.2 & 0 \\
0 & 0.2
\end{array}\right] \mathbf{B}^{2}
$$

- initial conditions:

$$
x_{0}=\left[\begin{array}{l}
0 \\
0
\end{array}\right], \hat{X}_{0}=\left[\begin{array}{l}
0 \\
0
\end{array}\right] \oplus\left[\begin{array}{ccc}
0.5 & 0 & 0 \\
0 & 0 & 0.5
\end{array}\right] \mathbf{B}^{3} .
$$

In the simulation, two faulty situations under the fault 1 and fault 2 are separately simulated. In both cases, the 
scenarios are set as: for the fault in the first or second sensor, from time instant 1 to 6 the system is healthy while at time instant 7 the first or second fault occurs.
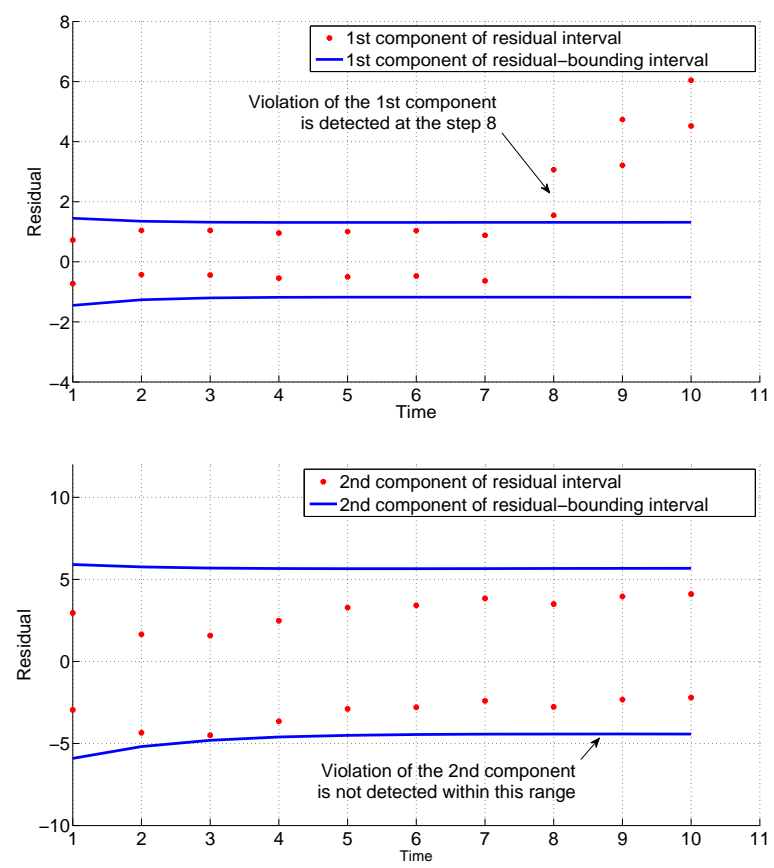

Fig. 2. FDI of the fault in the first sensor
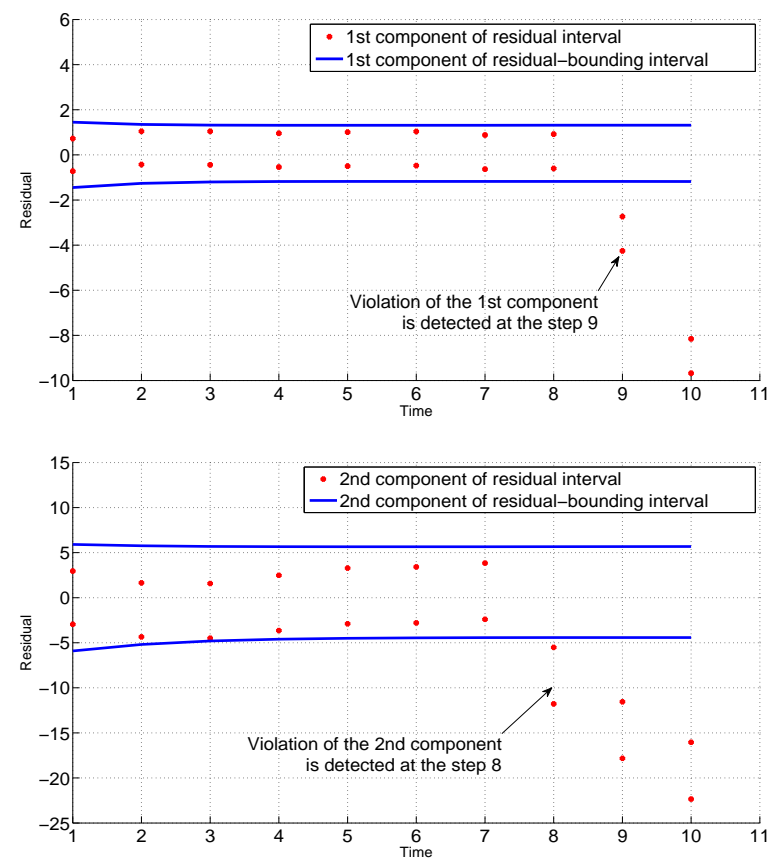

Fig. 3. FDI of the fault in the second sensor

Correspondingly, the FDI results of the faults in the two sensors are illustrated in Figure 2 and Figure 3. For the fault in the first sensor, comparing the two pictures in Figure 2, one can see that the fault can be accurately isolated.
Similarly, for the fault in the second sensor, comparing the two pictures in Figure 3, it is clearly seen that one step after the fault occurrence, the second component of the residual interval vector violates its threshold while the first component still respects its threshold, which implies that the fault is detected and isolated accurately and reliably.

However, one should emphasize that in Figure 3 more than one step after fault occurrence, the first component corresponding to the healthy sensor also violates its threshold. It is shown that the simulation results in Figure 2 and Figure 3 are consistent with the theoretical analysis presented in this paper, which presents the effectiveness of the proposed sensor FDI approach.

\section{CONCLUSIONS}

The paper proposes an interval observer-based sensor FDI approach, which defines the residuals in a novel way different from the classical residual definition of the interval observerbased FD approach. Each component of the residuals only corresponds to the fault in the corresponding sensor, which allows the proposed approach to detect and isolate sensor faults. The contribution of this paper mainly consists in that the proposed technique extends interval observers for sensor FI and can isolate faults one-step after fault occurrence. The future research is to define the isolable faults mathematically and establish guaranteed FDI conditions for this method.

\section{ACKNOWLEDGMENT}

The work of F. Xu, V. Puig and C. Ocampo-Martinez has been supported by Spanish research project WATMAN (CICYT DPI2009-13744) of the Science and Technology Ministry, by the DGR of Generalitat de Catalunya (SAC group Ref. 2009/SGR/1491), by European Comission through contract i-Sense (FP7-ICT-2009-6-270428), and by China Scholarship Council (File No.2011629170).

\section{REFERENCES}

[1] T. Alamo, J.M. Bravo, and E.F. Camacho. Guaranteed state estimation by zonotopes. In Proceedings of the 42nd IEEE Conference on Decision and Control, Maui, Hawaii, USA, December 2003.

[2] P. Guerra, V. Puig, and M. Witczak. Robust fault detection with unknown-input interval observers using zonotopes. In Proceedings of the 17th World Congress, The International Federation of Automatic Control, Coex, Seoul, South Korea, July 2008.

[3] J. Meseguer, V. Puig, T. Escobet, and R. Sarrate. Observer gain effect in linear interval observer-based fault detection. In the 46th IEEE Conference on Decision and Control, pages 995 -1002, dec. 2007.

[4] S. Olaru, J.A. De Doná, M.M. Seron, and F. Stoican. Positive invariant sets for fault tolerant multisensor control schemes. International Journal of Control, 83(12):2622-2640, 2010.

[5] V. Puig, J. Quevedo, T. Escobet, and A. Stancu. Passive robust fault detection using linear interval observers. In IFAC Safe Process, Washington, USA, 2003.

[6] F. Xu, F. Stoican, V. Puig, C. Ocampo-Martinez, and S. Olaru. Fault detection and isolation based on the combination of a bank of interval observers and invariant sets. In 21st Mediterranean Conference on Control and Automation, Platanias-Chania, Crete-Greece, June 2013. 